\title{
Results of Ultra-high Energy Cosmic Ray Study by Radio Technique at Yakutsk Array
}

\author{
Stanislav Knurenko*广 \\ $Y$ u. G. Shafer Institute of Cosmophysical Research and Aeronomy \\ E-mail: knurenko@ikfia.ysn.ru

\section{Igor Petrov} \\ Yu. G. Shafer Institute of Cosmophysical Research and Aeronomy \\ E-mail: igor.petrov@ikfia.ysn.ru
}

\begin{abstract}
Radio emission method is capable to study air shower physics independently from other shower components, including energy estimation of primary particle, longitudinal development of the particle cascade in the atmosphere (in the case of the Yakutsk array the depth of maximum development $\mathrm{X}_{\max }$ ), and thus, in the frame of chosen model, estimate mass composition of CR. Radio emission of air shower with energy $\mathrm{E} \sim 10^{20} \mathrm{eV}$ was first registered and its characteristics obtained at the Yakutsk array. For the showers with energy $\mathrm{E} \geq 10^{19} \mathrm{eV}$ arrival directions were tracked and reconstructed in galactic and equatorial coordinate system to search for search of CR sources with such energies.
\end{abstract}

36th International Cosmic Ray Conference -ICRC2019-

July 24th - August 1st, 2019

Madison, WI, U.S.A.

* Speaker.

${ }^{\dagger}$ A footnote may follow. 


\section{Introduction}

The method of registering radio emission from particles of air showers is based on the deflection of positrons and electrons by the Earth's magnetic field [1,2] and the Askaryan effect [3]. Apparently, both methods work effectively with the passage of a shower of particles through the atmosphere. Their contribution to the generation of radio emission depends on the conditions under which a shower develops in the atmosphere: the height of the maximum of the cascade curve, the zenith angle of arrival of the shower disk on the array plane, shower energy. Fully investigating these mechanisms of radio emission formation is available only on arrays with multiple detection technique of air shower particles: electrons, muons, Cherenkov light and radio emissions.

The radio method allows to estimate the energy, reconstruct longitudinal development of the shower, namely the depth of maximum development $X_{\max }[4,5]$. Using the maximum depth and energy, one can determine the type of primary particle that produced air shower in the atmosphere.

\section{Radio Array}

At the Yakutsk array, the frequency spectrum was scanned from 1 to $100 \mathrm{MHz}$ [6], as in [7], in order to select the optimal frequency range for radio emission detection. At frequencies up to $20 \mathrm{MHz}$, due to the presence of strong noises of natural origin (mainly thunderstorms), it is not possible to distinguish EAS impulses with sufficient efficiency. Above $20 \mathrm{MHz}$, the amplitude of galactic noise decreases much slower with a frequency than thunder noises and at a frequency of 32 $\mathrm{MHz}$ is $1.0-2.0 \mu \mathrm{Vm}^{-1} \cdot \mathrm{MHz}^{-1}$. The thermal noise of the antenna is much lower than galactic noise at frequencies up to $100 \mathrm{MHz}$ and almost does not affect our measurements in any way. Therefore, the optimal frequency band is $30-40 \mathrm{MHz}$, where the best signal-to-noise ratio is expected, because at higher frequencies the spectrum is limited by strong anthropogenic noises, such as broadcast stations. The array consists of 12 dual polarized antennas crossed under $90^{\circ}$, a peripheral recording device and an electronic data storage device based on a personal computer (Fig. 1). Antennas are located close to the center of the Yakutsk array and consisted two independent sets synchronized by the GPS. The distance between antennas are 50,100 and $500 \mathrm{~m}$. The antennas were located at a distance of 50-100 $\mathrm{m}$ from the observation station [8].

\section{Result}

\subsection{Radio Emission Amplitude Dependence of Energy}

In order to find a dependence of the amplitude of the radio emission $\varepsilon_{E W}$ on the shower energy $\mathrm{E}_{0}$ signal values were normalized to the median distance. Such normalization was due to the fact that the axes of most showers with radio emission were at a distance of 200-500 $\mathrm{m}[8,9]$. The energy of the showers was determined from the total flux of Cherenkov light by the energy balance method [10].

$$
\varepsilon_{E W}=(1.3 \pm 0.3)\left(\frac{E_{0}}{10^{17}} e V\right)^{0.99 \pm 0.04}
$$

Dependence of the amplitude on energy of air shower is shown in Fig. 2. 


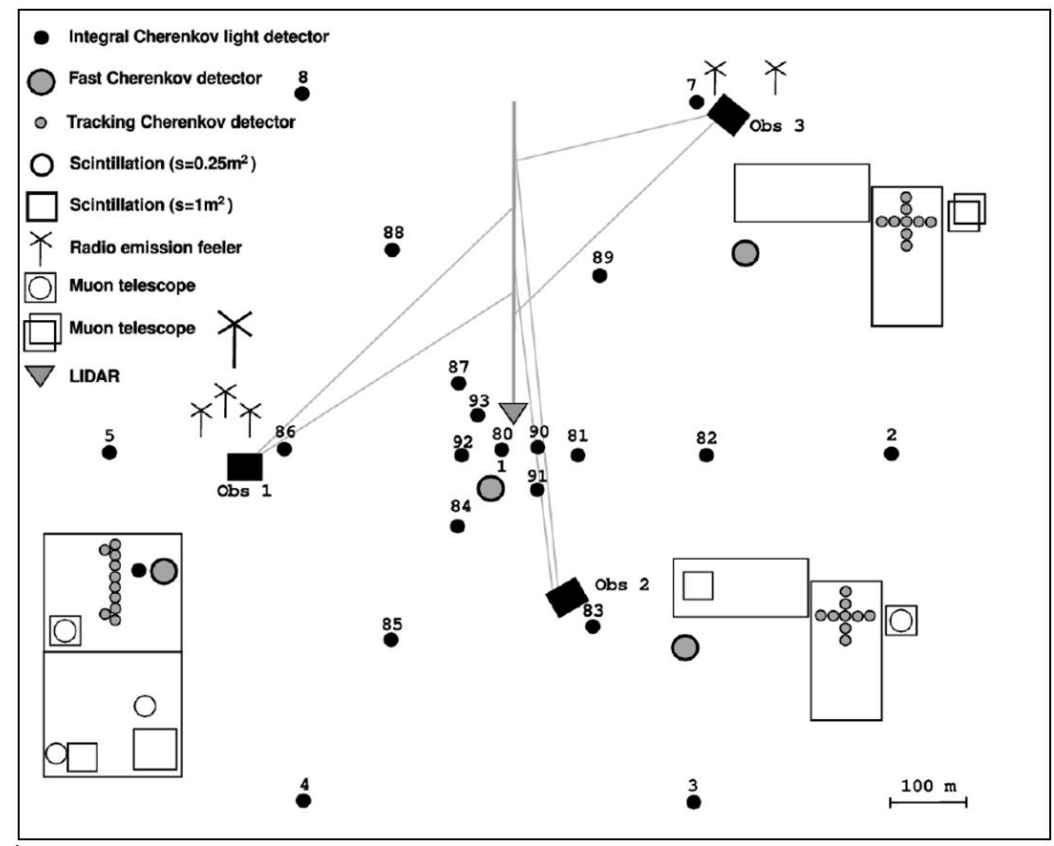

Figure 1: The layout of the antennas at the Yakutsk array

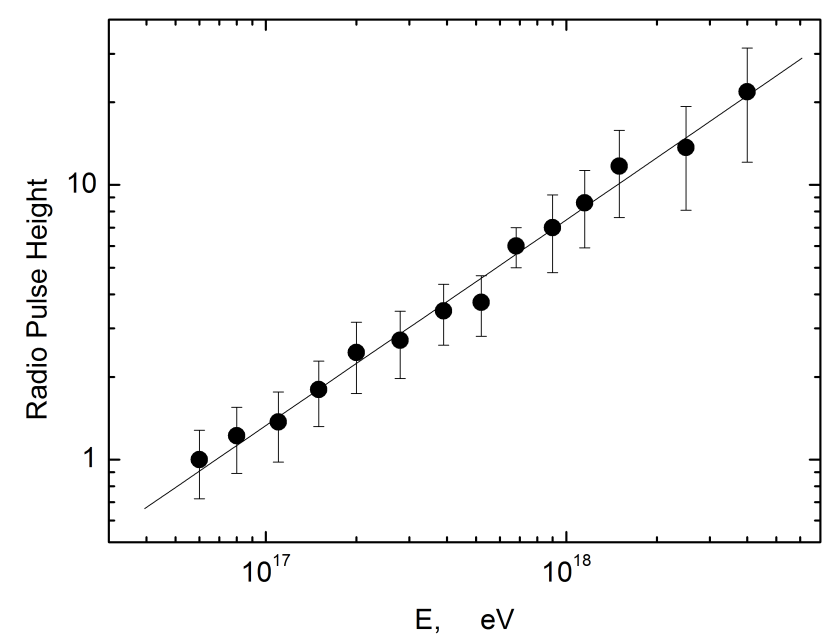

Figure 2: Dependence of the amplitude on energy of air shower 
The dots are the averaged amplitudes of the radio signal in a narrow bins over energy, and the line is an approximation from equation (1). From Fig. 2 it follows that there is a direct proportionality between the two characteristics of air showers. This dependence was used to estimate the energy of air showers by radio measurements, independently of the data of the main array.

\subsection{LDF Shape Dependence on Xmax}

As was shown in [9, 11-14], the function of the lateral distribution of radio emission reflects the longitudinal profile of the shower. According to the shape of the LDF, the depth of development maximum $\mathrm{X}_{\max }$ can be determined using the ratio of amplitudes at different distances from the shower axis $\mathrm{P}=\mathrm{A}_{1} / \mathrm{A}_{2}$ is used.

At the Yakutsk array the value of $\mathrm{P}=\mathrm{A}\left(\mathrm{R}_{1}\right) / \mathrm{A}\left(\mathrm{R}_{2}\right)$ correlation with $\mathrm{X}_{\max }$ was obtained empirically using simultaneous measurements of radio and Cherenkov emissions [8]:

$$
X_{\max }=(660 \pm 15)+(100 \pm 5) \cdot(P-11.5) / 3
$$

Formula (3.2) with sufficient accuracy is valid for the depth interval $\Delta X_{\max }=600-800 \mathrm{~g} \cdot \mathrm{cm}^{-2}$, below these depths, the uncertainty of $X_{\max }$ estimation increases.

Further, formula (3.2) was used mainly to determine $X_{\max }$ in individual showers with energies of $10^{17}-10^{18} \mathrm{eV}$. Using LDF of radio emission shape and formula (3.2) we obtained estimation of $X_{\text {max }}$ for different primary energies. The data of the Yakutsk array, obtained from measurements of Cherenkov light 1974-2014. (dots) and EAS radio emissions (triangles) are shown in Fig. 3a.

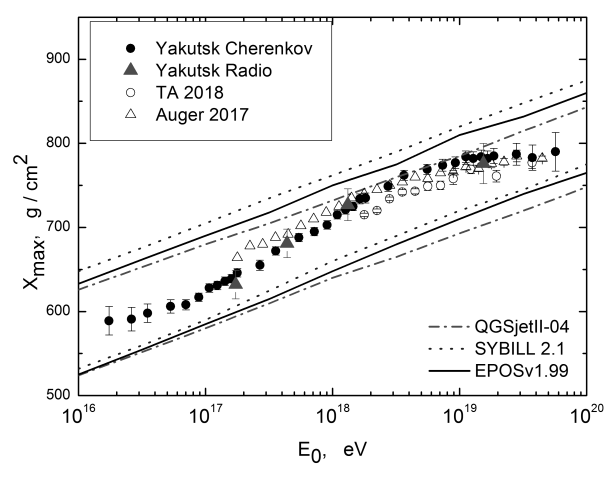

a)

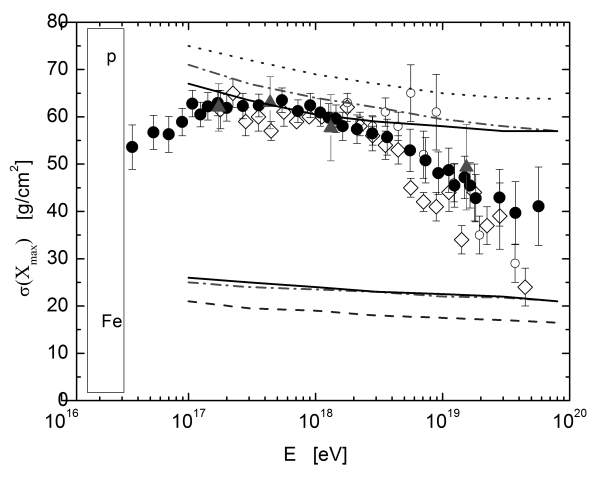

b)

Figure 3: (a) The dependence of Xmax on energy E0 obtained from observations of Cherenkov emission for the period 1974-2014 at the Yakutsk array (dots) and radio emission (triangles). Comparison with Auger (diamonds), Telescope Array (circles) and simulations. (b) Fluctuations of the depth of the maximum development of air showers in the energy range 3ï̌ĊU1016-5.7ấŹ1019 eV. Yakutsk Cherenkov data (dots), Yakutsk radio (triangles), Auger (diamonds) and TA (circles). Lines are simulations: QGSjetII-04 [17], Sybill 2.3c [18] and EPOS LHC [19] models for the primary proton and the iron nucleus.

Also, the comparison with the hybrid data $(\mathrm{BR} / \mathrm{LR}+\mathrm{SD})$ of the Telescope Array (circles) for 2018 [15], and Auger data presented at the ICRC 2017 (diamonds) [16] is shown in Fig. 3a. Also, simulations by the models of hadron interactions QGSJetII-04 [17], Sybill 2.3c [18] and EPOS LHC [19] are plotted. It can be seen that the experimental data of all arrays within the achieved 
accuracy are in good agreement with each other and indicate uneven advancement of $X_{\max }$ in the atmosphere. If we consider different energy intervals, we see that elongation rate (ER) has the following values of $48 \pm 6,78 \pm 5,63 \pm 6,50 \pm 7 \mathrm{~g} / \mathrm{cm}^{2}$. Such behavior of $X_{\max }$ most likely due to atomic weight change changes during the transition from one energy interval to another and this is due to the processes occurring at the sources and the interaction of the particles during the drift in the magnetic fields of cosmic medium. Comparison of experimental data with simulations also indicates a variable mass composition of the primary particles. In the energy range of $10^{16}-10^{17} \mathrm{eV}$, the composition most likely has more heavy nuclei, at energies of $10^{17}-10^{18} \mathrm{eV}$, the composition mainly consists of protons and light nuclei and above $10^{19} \mathrm{eV}$, the composition is enriched with heavy nuclei. This does not contradict the analysis of the velocity of displacement $X_{\max }$ in a wide energy range.

Thus, it can be concluded that by long-term observations at the Yakutsk array that the irregular development of air shower in the energy range $10^{16}-5.7 \cdot 10^{19} \mathrm{eV}$ in height, has been established.

To analyze the $X_{\max }$ fluctuations, a database of Cherenkov light of EAS for the period from 1970 to 2014 was used. Since the statistics of the showers allowed, the data array was divided into energy intervals with a step of 1.5 , and the value $\sigma\left(X_{\max }\right)$ was determined in each interval. Radio array data was divided into 4 energies in the range of $10^{17}-10^{19} \mathrm{eV}$. The results of the Yakutsk array are shown in Fig. 4. Radio emission data is also shown (triangles).

The data of other facilities and simulations of modern hadronic interaction models (Fig. 3b) for the primary proton $\mathrm{p}, \mathrm{CNO}$ nuclei and iron nucleus Fe are also displayed there. The experimental data of all the experiments within the statistical errors are consistent with each other, therefore it can be said that the obtained dependence of the fluctuations $X_{\max }$ is likely due to the mass composition of the primary particles that changes with energy. In the energy range of $10^{16}-10^{17} \mathrm{eV}$, the fluctuations of $X_{\max }$ are (50-60) $\mathrm{g} / \mathrm{cm}^{2}$ and tend to increase. In the energy region of $10^{17}-10^{18} \mathrm{eV}$, they are almost constant and above $10^{18} \mathrm{eV}$ decrease noticeably, reaching values of (50-40) $\mathrm{g} / \mathrm{cm}^{2}$.

Comparing the experimental data $\sigma\left(X_{\max }\right)$ with simulations for different nuclei, it can be said that the experimental results indicate a change in the mass composition. Qualitatively, it looks like this: in the region of lower energies, there are noticeably more nuclei with an atomic weight of $4-56$, at an energy of $10^{17}-10^{18} \mathrm{eV}$, the fraction of protons reaches a maximum and is $60-80 \%$, and then gradually decreases. In the energy region of $10^{19}-10^{20} \mathrm{eV}$ CR are composed of helium nuclei, $\mathrm{CNO}$ and heavier elements.

\subsection{Air Showers With Energy Higher Than $5 \cdot 10^{18} \mathbf{e V}$}

Fig. 4 shows the distribution of air showers with $E_{0} \geq 5 \cdot 10^{18} \mathrm{eV}$ on the sky map, coming one after another with an interval of less than 24 hours. The showers had similar characteristics: energy, zenith and azimuth angles. To some extent, they can be considered paired EAS, although the galactic coordinates in paired showers diverge by 10 degrees or more.

In the same place, crosses show showers with energies $E_{0} \geq 5 \cdot 10^{18} \mathrm{eV}$, registered by the Yakutsk radio array, during the observation periods of 1986-1989 and 2009-2018 Separately, triangles show showers with energy $\mathrm{E}_{0} \sim 10^{20} \mathrm{eV}$.

In Fig. 4 for comparison, the boundaries of the most active regions from which X-ray, radio and optical radiation are recorded are plotted: the constellation Ursa, Virgo, M82 and Markarian 421. In addition, a hot spot found according to TA data [20] is plotted. It can be seen that some 
of the showers coincide in their coordinates or are close to the boundaries of these regions, which indicates that active regions along with other sources may be sources of cosmic rays with ultra-high energies.

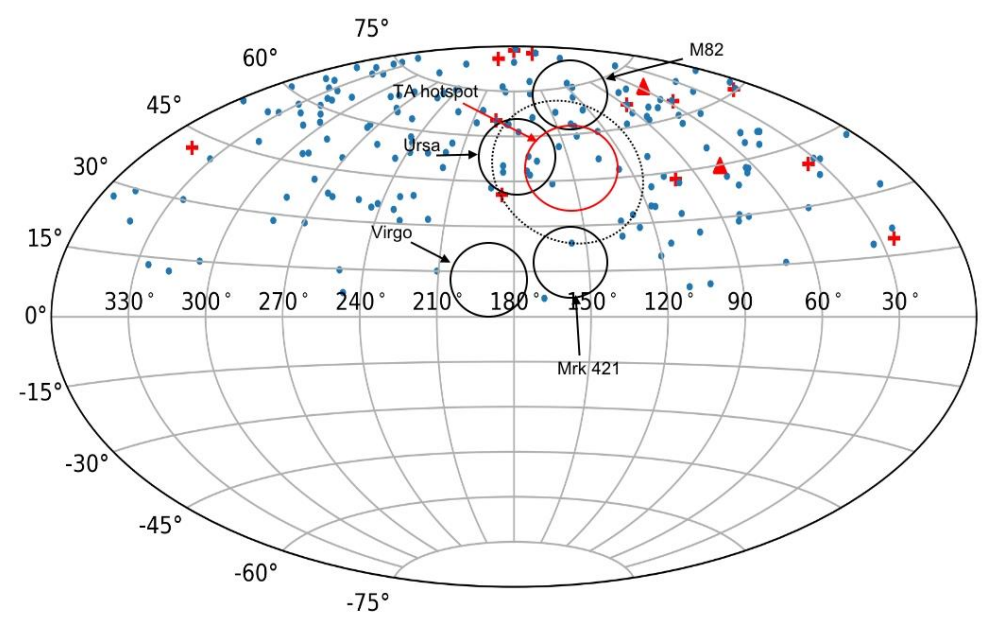

Figure 4: Distribution of the arrival of EAS events with $E_{0} \geq 5 \cdot 10^{18} \mathrm{eV}$

\section{Conclusion}

The obtained data on radio emission expands the possibilities of experimentally studying the characteristics of showers. As can be seen from Fig.3a and Fig. 3b, these results are in agreement with the data of other experiments. Using the data of Cherenkov detectors, the longitudinal development in the energy region $10^{16}-5.7 \cdot 10^{19} \mathrm{eV}$ was reconstructed and the dependence of $X_{\max }$ and $\sigma\left(X_{\max }\right)$ on energy was revealed. As can be seen from Fig. 3a, the advancement of $X_{\max }$ with increasing energy has an irregular course.

The offset rate $X_{\max }$ per decade of energy, elongation rate takes the values $48 \pm 6,78 \pm 5,63 \pm 6$, $50 \pm 7 \mathrm{~g} / \mathrm{cm}^{2}$. According to the measurement results, it can be said that in the region of lower energies, there are noticeably more nuclei with an atomic weight of $4-56$, at $10^{17}-10^{18} \mathrm{eV}$, the fraction of protons reaches a maximum and is $60-80 \%$, and then gradually decreases, and in the energy range $>10^{19} \mathrm{eV}$ cosmic rays consist of heavier elements.

Using database from 1986-1989 and 2009-2018, showers with energies above 5 ấź 1018 eV were selected and plotted on the sky map. Despite the low statistics, the data says that having a large area radio array, one can study the anisotropy of the arrival of showers by this method and search for sources generating particles of ultra-high energies.

\section{Acknowledgement}

The reported study was funded by RFBR according to the research project 16-29-13019. 


\section{References}

[1] F. Kahn and I. Lerche, Proc. of R. Soc. A. 289, 206 (1966).

[2] H. Falcke and P. Gorham, Astroparticle Physics, 19, 477 (2003).

[3] G.A. Askaryan, JETP, 41, 616 (1961).

[4] W.D. Apel et al., Phys. Rev. D., 90, 062001 (2014).

[5] S. Knurenko and I. Petrov, J. Phys.: Conf. Ser., 632, 012100 (2015).

[6] V. Kozlov et al., Proc. 1st Int. Conf. Electromagnetic Method of Environmental Studies, 215-217 (2012).

[7] S. Ellingson, J. Simonetti and C. Patterson, IEEE Trans. Antennas and Propag, 55, 826 (2007).

[8] S.P. Knurenko, Z.E. Petrov and I.S. Petrov, NIM A, 866, 230-241 (2017).

[9] S.P. Knurenko and I.S. Petrov, Bull. of the RAoS. Phys. 79, 410 (2015).

[10] S.P. Knurenko, A.A. Ivanov, I.E. Sleptsov and A.V. Sabourov, JETP Lett. 83, 563 (2006)

[11] T. Huege, R. Ulrich and R. Engel, Astropart. Phys., 30, 96 (2008).

[12] K.D. de Vries, A.M. van den Berg, O. Scholten and K. Werner, Astropart. Phys. 34, 267 (2010)

[13] N.N. Kalmykov and A.A. Konstantinov, J. Cosmol. Astropart. Phys. 12, 029 (2012)

[14] P.A. Bezyazeekov et al. J. Cosmol. Astropart. Phys. 01, 052 (2016).

[15] R.U. Abbasi et al. ApJ, 865, 74 (2018)

[16] A. Bellido et al. Proc. of Science 301, 506 (2018)

[17] S. Ostapchenko, Phys. Rev. D, 83, 014018 (2011).

[18] F. Riehn et al. Proc. of Science 236, 558 (2015).

[19] T. Pierog et al. Phys. Rev. C 92, 034906 (2015).

[20] J.N. Matthews et al. Proc. of Science, 301, 1096 (2018). 